\title{
BMJ Open User-centred clinical decision support to implement emergency department- initiated buprenorphine for opioid use disorder: protocol for the pragmatic group randomised EMBED trial
}

To cite: Melnick ER, Jeffery MM, Dziura JD, et al. User-centred clinical decision support to implement emergency department-initiated buprenorphine for opioid use disorder: protocol for the pragmatic group randomised EMBED trial. BMJ Open 2019;9:e028488. doi:10.1136/ bmjopen-2018-028488

- Prepublication history for this paper is available online. To view these files, please visit the journal online (http://dx.doi. org/10.1136/bmjopen-2018028488).

Received 12 December 2018 Revised 12 March 2019 Accepted 24 April 2019

Check for updates

(c) Author(s) (or their employer(s)) 2019. Re-use permitted under CC BY-NC. No commercial re-use. See rights and permissions. Published by BMJ.

For numbered affiliations see end of article.

Correspondence to

Dr Edward R Melnick;

edward.melnick@yale.edu

\section{ABSTRACT}

Introduction The goal of this trial is to determine whether implementation of a user-centred clinical decision support (CDS) system can increase adoption of initiation of buprenorphine (BUP) into the routine emergency care of individuals with opioid use disorder (OUD).

Methods A pragmatic cluster randomised trial is planned to be carried out in 20 emergency departments (EDs) across five healthcare systems over 18 months. The intervention consists of a user-centred CDS integrated into ED clinician electronic workflow and available for guidance to: (1) determine whether patients presenting to the ED meet criteria for OUD, (2) assess withdrawal symptoms and (3) ascertain and motivate patient willingness to initiate treatment. The CDS guides the ED clinician to initiate BUP and facilitate follow-up. The primary outcome is the rate of BUP initiated in the ED. Secondary outcomes are: (1) rates of receiving a referral, (2) fidelity with the CDS and (3) rates of clinicians providing any ED-initiated BUP, referral for ongoing treatment and receiving Drug Addiction Act of 2000 training. Primary and secondary outcomes will be analysed using generalised linear mixed models, with fixed effects for intervention status (CDS vs usual care), prespecified site and patient characteristics, and random effects for study site.

Ethics and dissemination The protocol has been approved by the Western Institutional Review Board. No identifiable private information will be collected from patients. A waiver of informed consent was obtained for the collection of data for clinician prescribing and other activities. As a minimal risk implementation study of established best practices, an Independent Study Monitor will be utilised in place of a Data Safety Monitoring Board. Results will be reported in ClinicalTrials.gov and published in open-access, peerreviewed journals, presented at national meetings and shared with the clinicians at participating sites via a broadcast email notification of publications.

Trial registration number NCT03658642; Pre-results.

\section{Strengths and limitations of this study}

- This pragmatic trial embeds a user-centred clinical decision support tool into routine care in the emergency department, reducing negative impact on providers and increasing the likelihood of immediate impact on actual care delivery.

- The streamlined workflow developed for the implementation of the intervention allows delivery across a large number of healthcare settings and across different electronic health records (EHR) systems, increasing the generalisability of the findings.

- Study length is shortened by the group-randomised design, better controlling for temporal trends in the opioid overdose epidemic.

- Constrained randomisation allows heterogeneity of included sites despite the small number of entities being randomised (a characteristic of group-randomised designs).

- Establishing patient eligibility for the intervention via phenotyping permits passive data collection from the EHR, reducing the reporting burden for sites.

\section{INTRODUCTION}

\section{Background and rationale}

Dependence on opioids is a major public health problem in the USA, taking a devastating toll on Americans, their families and communities. ${ }^{12}$ An estimated 2.1 million people in the USA have opioid use disorder (OUD) ${ }^{3}$ and more than 33000 opioid-related deaths occur annually. ${ }^{4}$ In 2011, there were 605000 emergency department (ED) visits related to opioids in the USA. ${ }^{5}$ From 2016 to 2017, EDs experienced a $30 \%$ increase in visits for opioid overdose. ${ }^{6}$ The ED offers a unique treatment opportunity for patients receiving care for acute and comorbid conditions related to opioid use. 
One of the most promising treatments for OUD is buprenorphine/naloxone (BUP), a partial opioid agonist combined with an antagonist, which can be prescribed by an appropriately trained clinician in an office setting for use at home. BUP decreases mortality as well as symptoms of withdrawal, craving and opioid use. ${ }^{78}$ In a placebo-controlled randomised trial of 40 OUD patients who all received cognitive-behavioural group therapy, weekly individual counselling and weekly urine drug screening, cumulative retention in treatment at 1 year was $75 \%$ for individuals in the BUP group compared with $0 \%$ in the placebo group $(p=0.0001){ }^{9}$ A recent Cochrane review including 31 trials with 5430 participants found highquality evidence that BUP is superior to placebo in retention of participants in treatment and can reduce illicit opioid use effectively compared with placebo. ${ }^{10}$

Currently, ED clinicians often refer patients with OUD to opioid treatment programmes rather than initiating medication for OUD (MOUD) treatment in the ED. In a randomised clinical trial involving 329 individuals with OUD, we found that ED-initiation of BUP with referral for ongoing MOUD treatment was superior to referral alone, resulting in nearly twice the percentage of patients who were engaged in formal addiction treatment at 30 days (78\% with BUP vs $37 \%$ with referral alone vs $45 \%$ with brief intervention, $\mathrm{p}<0.001$ ) and less illicit opioid use. ${ }^{11}$ Despite the efficacy of ED-initiated BUP with referral for ongoing MOUD treatment, it is currently not routinely offered in EDs due to medical, regulatory and logistical barriers. ${ }^{11-13}$ Adopting this evidence-based practice into routine care would shift the clinical practice paradigm for early OUD identification and treatment by initiating treatment at a time when the patient may be motivated and particularly vulnerable to morbidity and mortality. ${ }^{14}$

Clinical decision support (CDS), computerised tools that offer patient-specific assessments or recommendations to clinicians, represents one approach to embed this complex intervention into routine emergency care. ${ }^{16} 17$ However, CDS faces its own challenges, including unintended consequences, such as alert fatigue and increased cognitive load. ${ }^{18-22}$ CDS design recommendations suggest careful consideration of the sociotechnical environment and delivery of the right information, to the right person, in the right format, at the right time in clinical workflow to optimise medical decision-making. ${ }^{23-26}$

\section{Objectives}

For these reasons, we employed a user-centred design process to design and formatively evaluate the EMBED Emergency Department-Initiated Buprenorphine for Opioid Use Disorder) CDS intervention. The user-centred design and formative evaluation of the EMBED intervention is reported elsewhere. Given the current opioid epidemic in the USA, there is great urgency for prospective trials to identify the best approaches to BUP implementation and integration into routine practice. The goal of this multicentre, pragmatic, parallel, cluster randomised trial is to compare the effectiveness

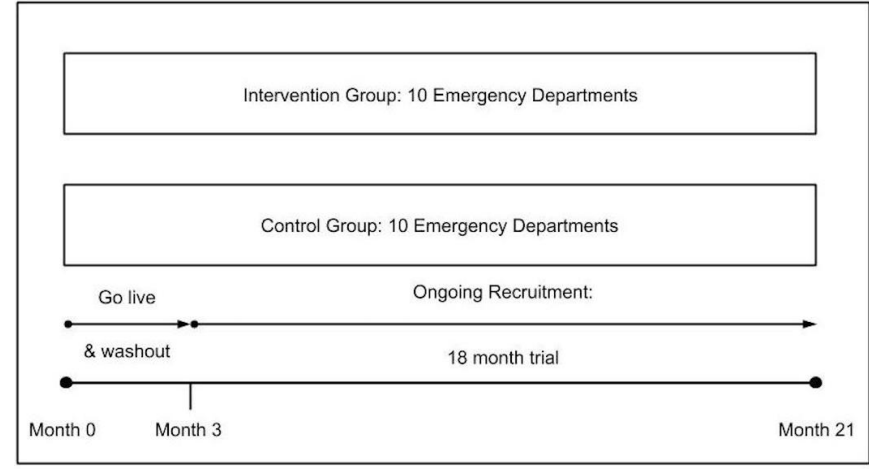

Figure 1 Schematic diagram of parallel, cluster randomised study design.

of user-centred CDS for ED-initiated BUP and referral for ongoing MOUD treatment to usual care on the rates of ED initiation of BUP and referral in ED patients with OUD. We hypothesise that rates of ED-initiation of BUP and referral will be higher in the user-centred CDS arm of the trial.

\section{Study design}

The study design is an 18-month pragmatic, parallel, cluster randomised, superiority trial using constrained randomisation of clusters to arms (schematic diagram, figure 1). ${ }^{27-29}$ The unit of randomisation (ie, cluster) is the ED. EDs will be randomly allocated with an allocation ratio of 1:1. Adequate lead time will be allotted to instal the intervention in the electronic health records (EHR) at all intervention sites-including a 3-month implementation and washout phase. The intervention will then begin at the same time across all sites with the CDS intervention fully implemented in the intervention sites' EHRs at the start of the trial. Clinicians at control sites will retain all control of their practice and practice as usual without the CDS intervention installed in their EHR.

Pragmatic trials study an intervention under the usual conditions in which it will be applied and generally use usual care as the comparator, as opposed to an explanatory trial, which would test an intervention under ideal conditions. ${ }^{27} 3031$ In cluster randomised trials, treatment intervention is allocated to clusters (ie, groups of individuals) rather than individuals. This is done to manipulate the physical or social environment of the intervention when an individual intervention would likely result in contamination between intervention and control participants at the group level. ${ }^{28}$ The parallel cluster randomised design was chosen over a stepped wedge design due to the high likelihood of confounding by temporal trends from ongoing efforts to mitigate the opioid epidemic. ${ }^{32} 33 \mathrm{~A}$ major challenge of the cluster randomised design is from potential confounding due to a limited number of heterogeneous groups. ${ }^{28}$ Constrained randomisation offers a solution to this source of confounding by balancing key cluster-level prognostic factors across the study to avoid distorting estimates of treatment effect due to the confounding factors. ${ }^{29}$ This allocation technique more 
evenly distributes potential confounders between intervention arms by specifying the confounding factors, characterising each cluster in terms of these factors, identifying a subset of randomisation combinations of clusters that adequately balance confounding factors between intervention arms and randomly selecting one of these combinations as the allocation scheme. ${ }^{29}$ Potential confounders that will be used for this trial are: EHR vendor, ED annual volume, ED type (eg, academic, community, urban, rural, etc), ratio of ED attendings who have a waiver to prescribe BUP, current rate of ED BUP prescribing, resources in ED to facilitate management of patients with OUD and willingness of staff to adopt the practice of ED initiation of BUP.

\section{METHODS}

\section{Participants}

There will be 20 participating EDs from hospitals in the USA within approximately five healthcare systems. At the time of writing this protocol, all of the sites have very low (or 0) rates of BUP initiation in the ED. The final study sites will be determined based on sample size needs, anticipated number of ED patients with OUD per site determined by EHR phenotype ${ }^{34-37}$ and willingness and ability to participate (eg, EHR integration of the intervention, EHR data extraction, availability of BUP in the $\mathrm{ED}$ and referral for ongoing MOUD treatment in the surrounding community). When finalised, the full studysite list will be available at ClinicalTrials.gov.

The intervention will be conducted at the site level. Patients are not considered human subjects since: (1) no identifiable private information will be collected, (2) the intervention does not target the patient and (3) EHR data will be collected retrospectively without interaction with the patient. The study sample will include all ED attending physicians credentialed to practice in the study site EDs. For ED encounters with physicians who practice at both an intervention and control site, only the encounters at intervention sites will contribute data for analyses. Encounters with these physicians at a control site will be excluded from the primary analysis.

Encounters with adult ED patients (aged 18 years or older) meeting an EHR-derived phenotype suggesting possible OUD will be included in the analysis: those who are discharged from the ED, not pregnant and not currently taking a MOUD. The initial phenotype has been developed by the study team and is currently undergoing validation via emergency physician chart review to determine the phenotype's validity in identifying the target patient population. ${ }^{34}$ Details of this phenotype and its validation will be reported separately. All encounters with ED patients meeting the EHR phenotype criteria will be eligible for the trial. For patients with more than one ED visit during the study period, only the initial ED visit will be eligible for inclusion in the primary analysis. The CDS will also be available for all clinicians on the care team and to use for encounters with patients who are not

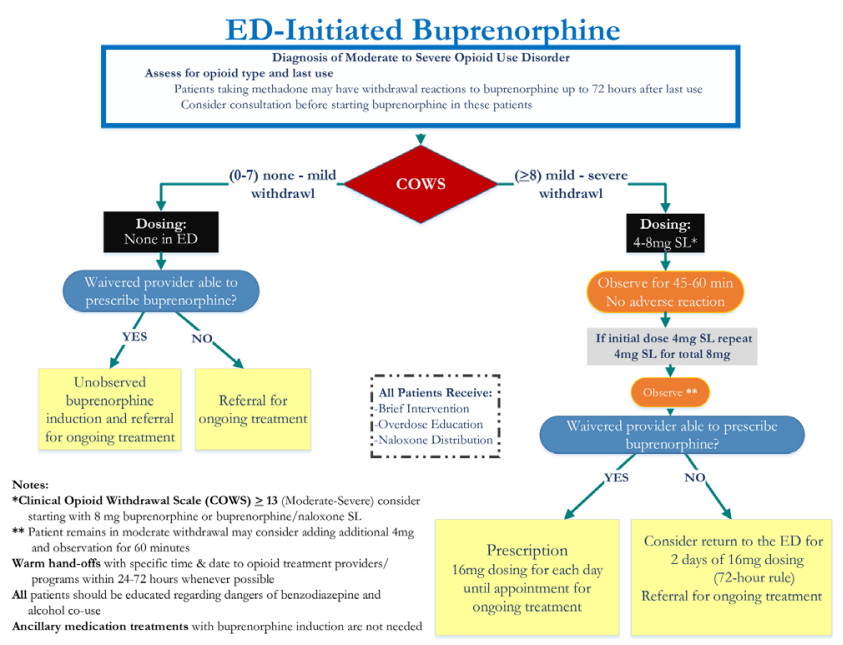

Figure 2 Clinical algorithm for ED initiation of buprenorphine. ED, emergency department; SL, sublingual.

identified by the phenotype. These encounters will be excluded from the primary analyses.

\section{Intervention}

The intervention for this study includes the user-centred CDS as well as education of ED clinicians practising at all study sites.

The need for flexibility in the graphical user interface of the intervention resulted in the decision to develop the CDS as a web application. This provides the ability to access the tool both embedded within the EHR or directly over the Internet. The web application was developed as a single-page application based on React JavaScript library. The CDS is a user-initiated activity in the EHR that calls the web application using Active Guidelines to streamline the flow diagram of our clinical protocol for ED-initiated BUP (figure 2). ${ }^{38}$

The intervention's graphical user interface (figure 3 ) is an intuitive, simple layout presenting four care pathways in columns based on the patient's diagnosis of OUD, the severity of withdrawal and readiness to start treatment. There is additional, optional decision support available for guidance to: (1) evaluate OUD severity based on Diagnostic and Statistical Manual of Mental Disorders, 5th Edition (DSM-5) criteria, (2) assess withdrawal severity using the Clinical Opiate Withdrawal Scale (COWS) score and (3) motivate patient willingness and readiness to initiate MOUD treatment with a brief motivational interview. ${ }^{39}$ These materials are also available to share with other members of the care team via a web address, text messaging or quick response $(\mathrm{QR})$ code. The interface also includes a toggle switch for the user based on whether or not they have a waiver to prescribe BUP. Non-waivered clinicians cannot prescribe BUP but can administer a one-time dose of BUP in the ED for up to 72 hours. ${ }^{41}$ When integrated into the local EHR system, launching a care pathway enables the user to: place 
TEXT 555-555-5.55

QR CODE

Select from one of the four treatment options below

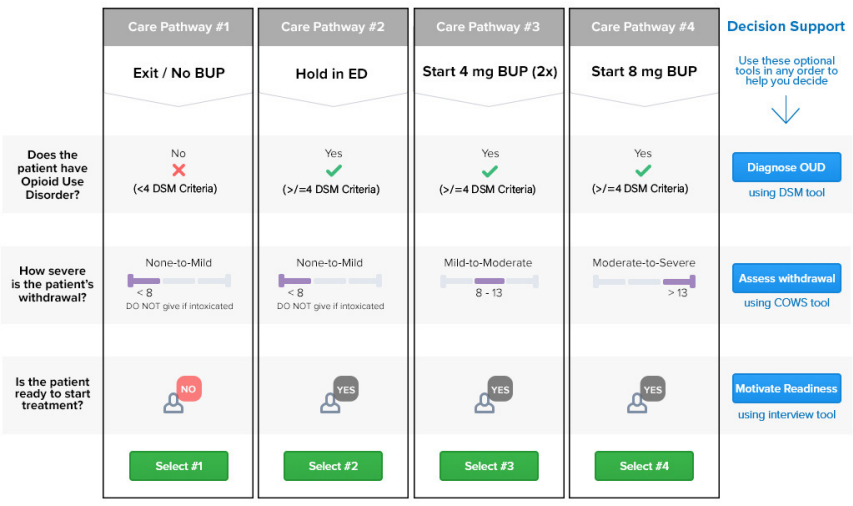

Figure 3 Graphical user interface of the user-centred CDS EMBED intervention. CDS, clinical decision support; COWS, Clinical Opiate Withdrawal Scale; ED, emergency department; EMBED, Emergency Department-Initiated Buprenorphine for Opioid Use Disorder; OUD, opioid use disorder.

orders, refer for ongoing MOUD treatment and update clinical notes.

The educational plan will be site-specific and tailored to the usual care at that institution. It will be administered within 3 months of the study start date. The details of the plan will be developed in partnership with local champions who self-identify an interest in helping to implement an ED-initiated BUP protocol at their site. Specifically, the education plan will be required to include:

1. A didactic on OUD, its diagnosis, assessment of withdrawal severity and local resources for referral for ongoing MOUD treatment.

2. Circulation and posting in each study site ED of the flow diagram of the study's clinical protocol for ED-initiated BUP (figure 2). Since this protocol is considered best practice, clinicians at control sites will retain all control of their practice and be encouraged to follow this protocol even though the CDS will not be available to them.

3. Intervention sites will include strategies to increase the use of the intervention by training clinicians on how to launch and use the CDS. Use of the intervention will be tracked with site-specific audit and feedback that is consistent with typical quality improvement initiatives at that site.

Given the ongoing and escalating opioid epidemic and a wide scope of this trial, we anticipate that there may be concomitant interventions to stem OUD at study sites during the trial. We plan to permit these interventions as long as they are: (1) implemented before randomisation so that they can be tracked and accounted for in the constrained randomisation process and (2) they are not a health information technology (IT) intervention targeted at clinicians to initiate BUP in the ED.

\section{Outcomes}

The primary study hypothesis is that there will be higher rates of ED-initiated BUP with referral for ongoing MOUD with user-centred CDS compared with usual care. Therefore, the primary outcome will be BUP initiation in the ED, defined as whether or not an eligible patient is administered BUP in the ED and/or prescribed BUP on discharge from the ED. Although this is not a patient-centred outcome, it is a pragmatic and meaningful surrogate that will serve as a lead indicator of the CDS intervention's effect on engaging more OUD patients in treatment.

We will also evaluate the effect of user-centred CDS on the following secondary implementation outcomes as compared with usual care, informed by the Reach, Effectiveness, Adoption, Implementation, Maintenance (RE-AIM) framework ${ }^{42}{ }^{43}$ :

1. Referral to follow-up for ongoing MOUD treatment made in the EHR (patient level; $\mathrm{Y} / \mathrm{N}$ ).

2. Prescription for naloxone at ED discharge (patient lev$\mathrm{el} ; \mathrm{Y} / \mathrm{N})$.

3. Receipt of discharge instructions on opioid use, overdose education, naloxone education and BUP education (patient level; $\mathrm{Y} / \mathrm{N}$ ).

4. Attending physician adoption rates (physician level): (a) provision of any ED-initiated BUP during the trial $(\mathrm{Y} / \mathrm{N})$ and $(\mathrm{b})$ provision of any referral for ongoing MOUD treatment during the trial $(\mathrm{Y} / \mathrm{N})$.

5. Receipt of Drug Addiction Treatment Act of 2000 training during trial (clinician level; $\mathrm{Y} / \mathrm{N}$ ).

Additional secondary implementation outcomes to be obtained from the web application include: clinician fidelity with the intervention assessed via a critical action checklist $^{44}$ and error rate of the intervention (using surrogates based on tool usage, for example, application launched but not used, launching a page in the web application and spending less than two seconds on that page). The intervention will continue to be made available for use after the trial concludes; 3 months after trial completion, medical record review of eligible patients will be conducted at a subset of intervention sites to determine the maintenance rate of the intervention.

\section{Sample size}

Current rates of BUP use in the ED range from $0 \%$ to $2 \%$ with most sites at $0 \%$. Assuming a rate of BUP use in the usual care group of $1 \%$, an increase to $10 \%$ would be a convincing and meaningful incremental effect of the intervention. Preliminary data from EDs that will be randomised in this trial suggest an intraclass correlation coefficient (ICC) for BUP use of 0.01. The National Institute of Health (NIH) group randomised sample size calculator ${ }^{45}$ was used to determine the required number of sites to be randomised. With a two-sided type I error of 0.05 , a conservative ICC of 0.03 and an expected average of 200 participants per site, a total of 12 sites will provide $90 \%$ power to detect a difference of $9 \%$. This estimate is based on the assumption that all sites will have at least 200 


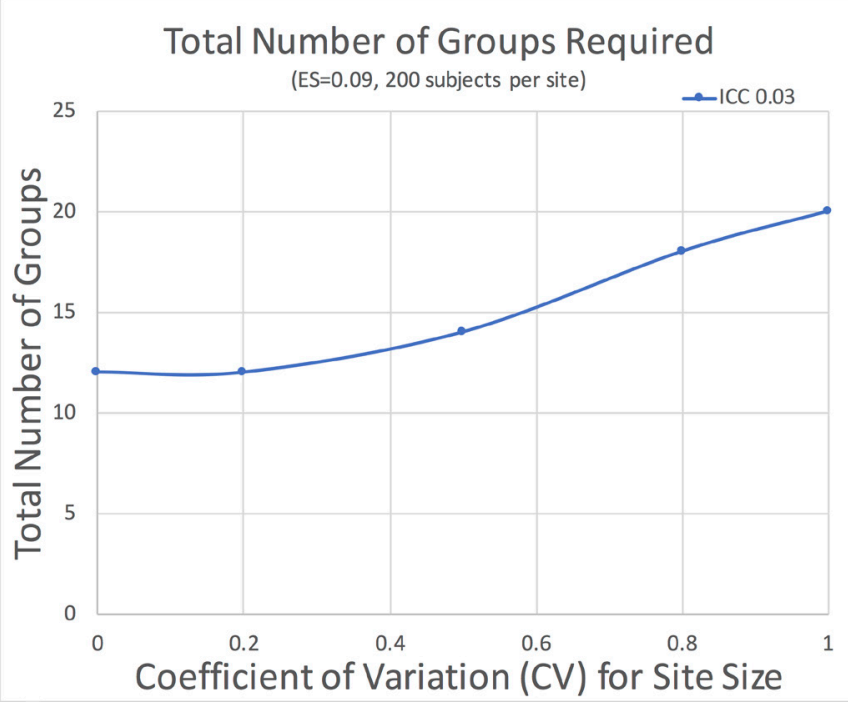

Figure 4 Number of study sites required as a function of coefficient of variation for site size assuming an ICC of 0.03. ICC, intraclass correlation coefficient.

unique patient visits during the trial that meet the EHR phenotype.

We estimated the impact of enrolment variability across sites on the required sample size using the formula described by Eldridge et $a l^{46}$ We added two sites to the total number of sites given the use of z-scores rather than $\mathrm{t}$-scores in the estimation. As the coefficient of variation in the number of participants enrolled across sites increases, the required number of sites increases (figure 4). To accommodate this potential variability, we will randomise a total of 20 sites (table 1 ).

\section{Allocation}

Study sites that meet readiness criteria at the time of randomisation will be allocated 1:1 to CDS and usual care groups using constrained randomisation conducted by personnel in the data coordinating centre (DCC). The general method will follow procedures and recommendations from the literature on group randomised trials. ${ }^{29}$

With a small number of sites that differ in important ways, unconstrained randomisation may not adequately balance important site characteristics. To improve comparability of treatment and control sites, personnel in the DCC under the direction of a senior statistician (JD) will list all possible allocations of treatment and control groups (with 20 sites, there are about 165000 combinations of treatment and control groups). The imbalance score $(\beta)$ from Raab and Butcher will be calculated for each possible allocation. ${ }^{47}$

$$
\beta=\sum_{l=1}^{S} \omega_{l}\left(\bar{x}_{0 l}-\bar{x}_{1 l}\right)^{2}
$$

where $S$ is the number of variables on which the groups should be balanced, $\omega_{l}$ is a weight calculated as the inverse variance of the mean of variable $l$ across the hospitals and the $\bar{x}_{l}$ represent the means of variable $l$ across the hospitals in the intervention (indexed as 1) and control (indexed as 0) groups. A candidate set of 1000 possible allocations with the most favourable imbalance scores will be selected, and the final allocation will be selected at random from that candidate set.

Since clinicians must know how to launch and use the intervention, they will not be blinded to the allocation of their site as a control or intervention site. Clinicians may inform patients that they are using the CDS or not, as they deem appropriate consistent with CDS use in their usual practice. All study sites will post information in their ED informing patients of the study.

\section{Data collection}

Outcome data will be collected via structured query language (SQL) query of the local EHR at regular intervals from data routinely collected in each hospital's EHR. This will facilitate large-scale data collection that would not otherwise be practical in an explanatory trial.

To enable consistent EHR data collection across sites, a master data dictionary of all data elements will be created. At each study site, the variables in the data dictionary will be validated against the institutional EHR to ensure that the variables are correctly mapped to the EHR field that corresponds to the clinical intent of the variable after accounting for documentation practices and workflow at each site. ${ }^{48}$ For data quality assurance, the mapped variables will be validated against the EHR to ensure that the data are clinically relevant to the goals of the project and correctly represents the clinical data that clinicians use to

Table 1 Power to detect different effect sizes (ES) by coefficient of variation (CV) in enrolment given randomisation of 20 sites

\section{Effect size (difference in proportions)}

\begin{tabular}{|c|c|c|c|c|c|c|}
\hline \multirow{5}{*}{$\begin{array}{l}\text { Coefficient of } \\
\text { variation in } \\
\text { enrolment }\end{array}$} & & 0.05 & 0.06 & 0.07 & 0.08 & 0.09 \\
\hline & 0 & $87 \%$ & $94 \%$ & $97 \%$ & $99 \%$ & $99 \%$ \\
\hline & 0.2 & $86 \%$ & $93 \%$ & $97 \%$ & $99 \%$ & $99 \%$ \\
\hline & 0.8 & $70 \%$ & $80 \%$ & $87 \%$ & $92 \%$ & $95 \%$ \\
\hline & 1.0 & $62 \%$ & $72 \%$ & $80 \%$ & $87 \%$ & $91 \%$ \\
\hline
\end{tabular}

Even with large variability in participant enrolment (CV=1), we will have over $90 \%$ power to detect a difference of 0.09 . We will have good power $(>80 \%)$ to detect effect sizes as low as 0.05 provided the variability in site enrolment is not great $(<0.50)$.

Grey represents power greater over $90 \%$, light grey represents power between $80-90 \%$, and medium grey represents power $<80 \%$. 
make decisions. Additionally, data to determine compliance, use and fidelity with the CDS intervention that could not be reliably abstracted from the EHR (eg, DSM-5 OUD score and COWS score) will be abstracted from the web application's use logs. Information on whether the patient attended the referred follow-up visit and whether the patient was prescribed BUP as an outpatient will be abstracted from the EHR only if available in the same EHR (eg, if the patient is seen for follow-up within the same system). Given the waiver of informed consent, we will be unable to track patients referred to out-of-system.

Data will be sent from study sites to the study DCC at predetermined, regular intervals: initially every 2 weeks, but adjusted as needed. The DCC will conduct ongoing data monitoring activities on study data from all participating sites to ensure data received is what it is intended to be. Baseline data for the study participants will include demographic and clinical data, such as age, gender, race, ethnicity, insurance status, past medical and psychiatric history, the most recent medical or psychiatric hospital admissions, recent enrolment in formal addiction treatment, active prescriptions for other opioids and urine drug screen results as ascertained by regularly collected data in the EHR.

\section{Data management}

Study data will only be available to members of the study DCC who are authorised for this study. To ensure the privacy and confidentiality of data for this project, DCC servers hosting data repositories are strongly firewalled; access to the repositories is permitted only through properly authenticated Web application program interface (APIs). All data will be encrypted both at rest and in transit. The DCC database hosting is certified by our institution's Information Security Office as conforming to Health Insurance Portability and Accountability Act of 1996 (HIPAA) and our institution's data protection guidelines. All project computers are stored in locked offices within a building having limited, electronic passkey access. All computers are password protected and protected by our institution's firewall, which is encrypted using Microsoft BitLocker. Individually identifiable or deducible data will only be by transmitted via secured telecommunications, never by unsecured telecommunications, such as email or electronic File Transfer Protocol. Procedures are in place for rapid recovery from hardware or database failure.

\section{Data monitoring}

As a minimal risk implementation study of established best practices, an Independent Study Monitor will be utilised in place of a formal Data Safety Monitoring Board. Interim monitoring will focus on adherence to the protocol, completeness of data retrieval from each ED's EHR and uptake of the CDS intervention. A set of monitoring tables will be generated for this purpose. The Independent Study Monitor will report directly to the study DCC. No interim analyses for effectiveness are planned.

\section{Study oversight}

As a UG3/UH3 Demonstration Project, the NIH Health Care Systems Research Collaboratory and its Steering Committee core serve as the coordinating centre and steering committee for this project and have given ongoing support to the design and rapid execution of this project. The DCC is composed of two biostatisticians, two clinical informaticists, a database manager and two computer programmers. Study progress will be audited monthly for the first 3 months of the trial and then quarterly (but more frequently if needed) independent from investigators and the sponsor. Progress reports, including study progress and any adverse events, will be provided to the Independent Study Monitor following each of the monthly reviews. In terms of progress, auditing will focus on adherence to the protocol, completeness of data retrieval from each ED's EHR and uptake of the CDS intervention. A set of monitoring tables will be generated by the DCC for this purpose.

\section{Analysis plan}

General Considerations: This is a cluster randomised trial to test the hypothesis that there will be higher rates of provision of ED-initiated BUP and referral for ongoing MOUD with user-centred CDS compared with usual care. Analyses will be conducted as intention-to-treat including all individuals regardless of intervention receipt. While the unit of randomisation is at the level of the ED, the unit of analysis will be the patient. Analyses of primary and secondary outcomes will be conducted using logistic regression with weighted generalised estimating equations (GEE) to account for clustering from the EDs and physicians in patient outcome models. ${ }^{49}$ Analyses will be performed in SAS V.9.4 with a two-sided type I error of 0.05 (unless otherwise specified). For the primary and secondary analyses described below, only the first ED encounter for an individual patient will be used. Supportive analyses will include patients with repeated ED visits.

Comparability of Baseline and Intervention Site Patients: Distributions of baseline demographic and clinical characteristics will be described during baseline and intervention periods. Comparability for continuous variables will be examined graphically and by summary statistics (means, medians, quartiles, and so on). Categorical variables will be examined by calculating frequency distributions.

Analysis of Primary Outcome: The primary outcome, initiation of BUP in the ED, will be assessed for all patients that meet the criteria for the EHR phenotype. Intervention differences (CDS vs usual care) for this dichotomous outcome will be examined using weighted GEE. The weighted GEE provides consistent parameter estimates when the dropout mechanism is correctly classified by implementing the inverse-probability weighted method to account for dropouts under the missing at random (MAR) assumption. Inverse-probability weights are estimated by a logistic regression of dropout. The weighted GEE model will contain an effect for intervention (CDS 
vs usual care). An exchangeable working correlation will be used to account for clustering of responses within ED and physicians. The model will also include cluster-level covariates included in the constrained randomisation and patient-level covariates that may be associated with the delivery of BUP (age, gender, race, ethnicity, insurance status, medical and psychiatric history, recent medical or psychiatric hospital admissions, recent enrolment in formal addiction treatment, active prescriptions for other opioids and urine drug screen results). Linear contrasts will be used to estimate treatment differences along with $95 \%$ CIs in the proportions of ED patients that received BUP in CDS versus usual care. Given the relative advantages of GEE and generalised linear mixed models (GLMM), sensitivity analyses will compare treatments using a logistic regression with GLMM, with random effects for ED and physician.

Analysis of Secondary Outcomes: Secondary outcomes, such as referral for MOUD appointment, attendance at an MOUD appointment (if available in the EHR), prescription for naloxone at ED discharge and receipt of discharge instructions will be evaluated using weighted GEE as described above. Assessments of the physician including provision of any ED-initiated BUP during the trial, provision of any referral for ongoing MOUD treatment during the trial and receipt of Drug Addiction Treatment Act of 2000 training during the trial will be compared between CDS and usual care using GEE. These models will be stratified by the number of eligible patients the physician encountered during the trial and will include an effect for intervention, cluster-level covariates included in the constrained randomisation, and an exchangeable working correlation. Discrete numeric outcomes, such as clinical fidelity, will be compared using the GEE with a log link and a negative binomial distribution.

Plan for Missing Data: Several strategies will be imposed to accommodate the likelihood that missing data will occur during this study. Prevention is the most obvious and effective manner to control bias and loss of power from missing data. ${ }^{50}$ As noted in the section Data collection, prior to the trial, we will pilot data collection procedures. Variables with large proportions of missing will be excluded from the collection. We will follow the intent-totreat principle, requiring follow-up of all EDs randomised regardless of the treatment received. ${ }^{51}$ Regular data retrieval from EHRs combined with monitoring and missing data reports will trigger protocols for tracking and obtaining missing data. Despite these prevention efforts, it is reasonable to assume missing data will occur. Our primary analysis is valid under the assumption that missing data will be MAR. ${ }^{52}$ We will evaluate the plausibility of this assumption by determining the extent of missing data and use logistic regression to identify factors associated with missing data. As appropriate, we will conduct sensitivity analysis using pattern-mixture and selection models under missing not at random assumptions to examine the robustness of conclusions of the primary analysis to missing data. ${ }^{50} 52$

\section{Patient and public involvement}

The development of the research question and outcome measures was led by emergency physicians, the primary population being studied. Formal user design sessions were conducted with both attending and resident physicians to ensure that the CDS would be useful and would not interfere with patient care nor pose an undue burden on clinicians' time. The results of the study will be shared with the clinicians at participating sites via a broadcast email notification of publications. Patients did not participate in the design of the study and will not be involved in the recruitment and conduct of the study.

\section{ETHICS AND DISSEMINATION}

We plan to obtain all necessary regulatory and human subjects protection approvals and procedures. Any important protocol modifications will be submitted to the Western Institutional Review Board as protocol amendments. The local IRBs at each participating site will implement a reliance agreement with this central board. We anticipate a waiver of informed consent under the Common Rule (45 code of federal regulations (CFR) 46.116 given that ${ }^{53} 54$ : (1) the research involves no more than minimal risk to the subjects ${ }^{55}$; $(2)$ the waiver or alteration will not adversely affect the rights and welfare of the subjects; (3) the research could not practicably be carried out without the waiver or alteration and (4) subjects will be provided with additional pertinent information after participation.

Patients are not considered human subjects by Health \& Human Services (HHS) regulation 45 CFR 46.102(f) ${ }^{54}$ since: (1) no identifiable private information will be collected, (2) the intervention does not target the patient and (3) EHR data will be collected retrospectively without interaction with the patient. Furthermore, all recommendations included in the CDS intervention are considered best practices in treatment of OUD. The OUD population has a high underlying risk of morbidity/mortality (approximately 5\% risk of death in 12 months). ${ }^{8}$ Patient rights and welfare will be protected per standard practice. Therefore, the risk to a patient with OUD who is not receiving MOUD treatment in their ordinary daily lives greatly exceeds the risk of the EMBED intervention. All study sites will post details about the study in a location visible to patients to make them aware of the option to receive BUP and referral to treatment so as best to offer an informed decision for requesting care. Patients will retain the right to request MOUD treatment at any study site.

Clinicians at all study sites will have access to all standard OUD medications and services to which they would otherwise have access to treat OUD patients. Clinicians will retain all control of their practice and at intervention sites have the option whether or not to use the intervention (ie, can opt out). Clinician identifiers will be collected in order to follow practice patterns. However, the investigators will be blinded to both site and clinician identifiers. 
Each system will use an Honest Broker to protect the welfare and identity of each site and clinician, and allow adjudication for analyses. Clinicians will be made aware of the study, its outcomes, the data to be collected and, at intervention sites, how to use and opt out of using the CDS via broadcast email and direct communication by site champions. A flow diagram of the study's clinical protocol (figure 2) will be shared with clinicians and posted in the clinical work area of all study sites. Since this protocol is considered best practice, clinicians at control sites will retain all control of their practice and be encouraged to follow this protocol even though the CDS will not be available to them. As this is a pragmatic trial focused on implementing this intervention in a way that is as close to routine care as possible, consenting clinicians would not be consistent with routine CDS implementation and could jeopardise the scientific validity of the CDS intervention to overcome barriers to adoption of this practice.$^{53}$ Given the stigma ${ }^{11}$ associated with treating individuals with OUD, the additional burden of the consent process could be a deterrent for clinicians to provide MOUD treatment to appropriate patients and bias the sample to clinicians with less stigma toward treating these patients. For this reason and since clinician data will be de-identified and unavailable to the investigators, we propose a waiver of consent of the clinicians to ensure the scientific validity of our findings. There is a precedent for such a waiver in a similar situation. ${ }^{56}$

Results will be published in open-access, peer-reviewed journals, presented at national meetings and shared with the clinicians at participating sites via a broadcast email notification of publications. The full protocol will be published for public access; access to the participant-level data set will be made in accordance with NIH policy after safeguarding that the data sets are fully de-identified at the site, provider and patient level. No professional writers will be used. Authorship eligibility will follow the guidelines of the International Committee of Medical Journal Editors. ${ }^{57}$

\section{Author affiliations}

${ }^{1}$ Department of Emergency Medicine, Yale University School of Medicine, New Haven, Connecticut, USA

${ }^{2}$ Department of Emergency Medicine, Mayo Clinic, Rochester, Minnesota, USA ${ }^{3}$ Emergency Medicine, Eastern Virginia Medical School, Norfolk, Virginia, USA ${ }^{4}$ Department of Emergency Medicine, University of Alabama at Birmingham School of Medicine, Birmingham, Alabama, USA

${ }^{5}$ Department of Emergency Medicine, University of North Carolina School of Medicine, Chapel Hill, North Carolina, USA

${ }^{6}$ Information Technology Services, Yale New-Haven Health, New Haven, Connecticut, USA

${ }^{7}$ Department of Emergency Medicine, University of North Carolina at Chapel Hill School of Medicine, Chapel Hill, North Carolina, USA

${ }^{8}$ Yale Center for Medical Informatics, Yale University School of Medicine, New Haven, Connecticut, USA

Contributors ERM and GD conceived of the work. ERM, MMJ, JDD, EPH, TFP-M and GD designed the study. ERM, MMJ, EPH, TFP-M, YS, HP, SM, MDP, CL, CB and $\mathrm{GD}$ are responsible for the implementation of the trial. ERM, MMJ, JAM, JDD (sample size, allocation and analysis) and LB (ethics) drafted the initial manuscript.
All authors edited and approved the final version submitted for publication. ERM takes responsibility for all aspects of the work.

Funding This work is supported within the NIH Health Care Systems Research Collaboratory, by a cooperative agreement (UG3DA047003) from the National Institute on Drug Abuse of the NIH. This work also received logistical and technical support from the NIH Collaboratory Coordinating Center (U24AT009676). Funder contact information: National Institute on Drug Abuse, Office of Science Policy and Communications, Public Information and Liaison Branch, 6001 Executive Boulevard, Room 5213, MSC 9561, Bethesda, MD 20892, USA.

Disclaimer The content is solely the responsibility of the authors and does not necessarily represent the official views of the National Institutes of Health. The funders had no role in the decision to publish or the preparation of the manuscript. The National Institutes of Health contributed to the design of the study.

Competing interests None declared.

Patient consent for publication Not required.

Ethics approval The protocol has been approved by the Western Institutional Review Board, central IRB (protocol number 20182278, study number 1189765).

Provenance and peer review Not commissioned; externally peer reviewed.

Open access This is an open access article distributed in accordance with the Creative Commons Attribution Non Commercial (CC BY-NC 4.0) license, which permits others to distribute, remix, adapt, build upon this work non-commercially, and license their derivative works on different terms, provided the original work is properly cited, appropriate credit is given, any changes made indicated, and the use is non-commercial. See: http://creativecommons.org/licenses/by-nc/4.0/.

\section{REFERENCES}

1. Rudd RA, Aleshire N, Zibbell JE, et al. Increases in drug and opioid overdose deaths--United States, 2000-2014. MMWR Morb Mortal Wkly Rep 2016;64:1378-82.

2. Buchanich JM, Balmert LC, Burke DS. Exponential Growth Of The USA Overdose Epidemic. bioRxiv. 2017 https://www.biorxiv.org/ content/early/2017/05/09/134403.abstract.

3. Center for Behavioral Health Statistics and Quality. Key Substance Use and Mental Health Indicators in the United States: Results from the 2015 National Survey on Drug Use and Health. Substance Abuse and Mental Health Services Administration. 2016 https://www. samhsa.gov/data/sites/default/files/NSDUH-FFR1-2015/NSDUHFFR1-2015/NSDUH-FFR1-2015.htm.

4. Seth P, Scholl L, Rudd RA, et al. Overdose deaths involving opioids, cocaine, and psychostimulants - United States, 2015-2016. MMWR Morb Mortal Wkly Rep 2018;67:349-58.

5. Substance Abuse and Mental Health Services Administration. Drug Abuse Warning Network. 2011: National Estimates of Drug-Related Emergency Department Visits. 2013 https://www.samhsa.gov/data/ sites/default/files/DAWN2k11ED/DAWN2k11ED/DAWN2k11ED.pdf.

6. Vivolo-Kantor AM, Seth P, Gladden RM, et al. Vital signs: trends in emergency department visits for suspected opioid overdoses United States, July 2016-September 2017. MMWR Morb Mortal Wkly Rep 2018;67:279-85.

7. Sullivan LE, Fiellin DA. Narrative review: buprenorphine for opioid-dependent patients in office practice. Ann Intern Med 2008;148:662-70.

8. Larochelle MR, Bernson D, Land T, et al. Medication for opioid use disorder after nonfatal opioid overdose and association with mortality: a cohort study. Ann Intern Med 2018;169.

9. Kakko J, Svanborg KD, Kreek MJ, et al. 1-year retention and social function after buprenorphine-assisted relapse prevention treatment for heroin dependence in Sweden: a randomised, placebo-controlled trial. Lancet 2003;361:662-8.

10. Mattick RP, Breen C, Kimber J, et al. Buprenorphine maintenance versus placebo or methadone maintenance for opioid dependence. Cochrane Database Syst Rev 2014:CD002207.

11. D'Onofrio G, O'Connor PG, Pantalon MV, et al. Emergency department-initiated buprenorphine/naloxone treatment for opioid dependence: a randomized clinical trial. JAMA 2015;313:1636-44.

12. Goodnough A. This E.R. Treats Opioid Addiction on Demand. That's Very Rare. https://www.nytimes.com/2018/08/18/health/opioidaddiction-treatment.html. (accessed 2 May 2019).

13. Walsh L. Buprenorphine Waiver Management | SAMHSA - Substance Abuse and Mental Health Services Administration. https://www. samhsa.gov/programs-campaigns/medication-assisted-treatment/ 
training-materials-resources/buprenorphine-waiver (accessed $17 \mathrm{Jul}$ 2018).

14. Duber HC, Barata IA, Cioè-Peña E, et al. Identification, management, and transition of care for patients with opioid use disorder in the emergency department. Ann Emerg Med 2018;72.

15. Houry DE, Haegerich TM, Vivolo-Kantor A. Opportunities for prevention and intervention of opioid overdose in the emergency department. Ann Emerg Med 2018;71:688-90.

16. Kawamoto K, Houlihan CA, Balas EA, et al. Improving clinical practice using clinical decision support systems: a systematic review of trials to identify features critical to success. BMJ 2005;330:765

17. Garg AX, Adhikari NK, McDonald H, et al. Effects of computerized clinical decision support systems on practitioner performance and patient outcomes: a systematic review. JAMA 2005;293:1223-38.

18. Sittig DF, Wright A, Osheroff JA, et al. Grand challenges in clinical decision support. J Biomed Inform 2008;41:387-92.

19. Ash JS, Berg M, Coiera E. Some unintended consequences of information technology in health care: the nature of patient care information system-related errors. J Am Med Inform Assoc 2004;11:104-12.

20. Ash JS, Sittig DF, Campbell EM, et al. Some unintended consequences of clinical decision support systems. AMIA Annu Symp Proc 2007:26-30.

21. Levin S, France DJ, Hemphill R, et al. Tracking workload in the emergency department. Hum Factors 2006;48:526-39.

22. Melnick ER, Nielson JA, Finnell JT, et al. Delphi consensus on the feasibility of translating the ACEP clinical policies into computerized clinical decision support. Ann Emerg Med 2010;56:317-20.

23. Sirajuddin AM, Osheroff JA, Sittig DF, et al. Implementation pearls from a new guidebook on improving medication use and outcomes with clinical decision support. Effective CDS is essential for addressing healthcare performance improvement imperatives. $J$ Healthc Inf Manag 2009;23:38-45.

24. Phansalkar S, Edworthy J, Hellier E, et al. A review of human factors principles for the design and implementation of medication safety alerts in clinical information systems. J Am Med Inform Assoc 2010;17:493-501.

25. Horsky J, Phansalkar S, Desai A, et al. Design of decision support interventions for medication prescribing. Int J Med Inform 2013;82:492-503.

26. Gellert G, Webster L, Gillean J, et al. Should US doctors embrace electronic health records? BMJ 2017;356:j242.

27. Roland $\mathrm{M}$, Torgerson DJ. What are pragmatic trials? BMJ 1998;316:285

28. Murray DM, Varnell SP, Blitstein JL. Design and analysis of grouprandomized trials: a review of recent methodological developments. Am J Public Health 2004;94:423-32.

29. Li F, Lokhnygina Y, Murray DM, et al. An evaluation of constrained randomization for the design and analysis of group-randomized trials. Stat Med 2016;35:1565-79.

30. Loudon K, Treweek S, Sullivan F, et al. The PRECIS-2 tool: designing trials that are fit for purpose. BMJ 2015;350:h2147.

31. Zuidgeest MGP, Welsing PMJ, van Thiel G, et al. Series: Pragmatic trials and real world evidence: Paper 5. Usual care and real life comparators. J Clin Epidemiol 2017;90:92-8.

32. Hussey MA, Hughes JP. Design and analysis of stepped wedge cluster randomized trials. Contemp Clin Trials 2007;28:182-91.

33. Hemming K, Haines TP, Chilton PJ, et al. The stepped wedge cluster randomised trial: rationale, design, analysis, and reporting. BMJ 2015;350:h391.

34. Richesson RL, Hammond WE, Nahm M, et al. Electronic health records based phenotyping in next-generation clinical trials: a perspective from the NIH Health Care Systems Collaboratory. J Am Med Inform Assoc 2013;20:e226-31.

35. Hylan TR, Von Korff M, Saunders K, et al. Automated prediction of risk for problem opioid use in a primary care setting. $J$ Pain 2015;16:380-7.
36. Carrell DS, Cronkite D, Palmer RE, et al. Using natural language processing to identify problem usage of prescription opioids. Int $J$ Med Inform 2015;84:1057-64.

37. Reardon JM, Harmon KJ, Schult GC, et al. Use of diagnosis codes for detection of clinically significant opioid poisoning in the emergency department: A retrospective analysis of a surveillance case definition. BMC Emerg Med 2016;16:11.

38. Yale School of Medicine. ED-Initiated Buprenorphine | Emergency Medicine. https://medicine.yale.edu/edbup/ (accessed 24 Aug 2018).

39. American Psychiatric Association. Diagnostic and statistical manual of mental disorders (DSM-5®). American Psychiatric Pub 2013.

40. Wesson DR, Ling W. The Clinical Opiate Withdrawal Scale (COWS). J Psychoactive Drugs 2003;35:253-9.

41. Walsh L. Special Circumstances for Providing Buprenorphine I SAMHSA - Substance Abuse and Mental Health Services Administration. https://www.samhsa.gov/programs-campaigns/ medication-assisted-treatment/legislation-regulations-guidelines/ special (accessed 13 Aug 2018).

42. Glasgow RE, Vogt TM, Boles SM. Evaluating the public health impact of health promotion interventions: the RE-AIM framework. Am J Public Health 1999;89:1322-7.

43. Glasgow RE, Lichtenstein E, Marcus AC. Why don't we see more translation of health promotion research to practice? Rethinking the efficacy-to-effectiveness transition. Am J Public Health 2003;93:1261-7.

44. LeBlanc A, Ruud KL, Branda ME, et al. The impact of decision aids to enhance shared decision making for diabetes (the DAD study): protocol of a cluster randomized trial. BMC Health Serv Res 2012;12:130.

45. National Institutes of Health (NIH). Research methods resources. https://researchmethodsresources.nih.gov/SampleSizeCalculator. aspx (accessed 13 Aug 2018)

46. Eldridge SM, Ashby D, Kerry S. Sample size for cluster randomized trials: effect of coefficient of variation of cluster size and analysis method. Int J Epidemiol 2006;35:1292-300.

47. Raab GM, Butcher I. Balance in cluster randomized trials. Stat Med 2001;20:351-65.

48. Gong MN, Schenk L, Gajic O, et al. Early intervention of patients at risk for acute respiratory failure and prolonged mechanical ventilation with a checklist aimed at the prevention of organ failure: protocol for a pragmatic stepped-wedged cluster trial of PROOFCheck. BMJ Open 2016:6:e011347.

49. Preisser JS, Lohman KK, Rathouz PJ. Performance of weighted estimating equations for longitudinal binary data with drop-outs missing at random. Stat Med 2002;21:3035-54.

50. National Research Council, Division of Behavioral and Social Sciences and Education, Committee on National Statistics. The prevention and treatment of missing data in clinical trials: National Academies Press, 2010.

51. Lachin JM. Statistical considerations in the intent-to-treat principle. Control Clin Trials 2000;21:167-89.

52. Molenberghs $\mathrm{G}$, Thijs $\mathrm{H}$, Jansen $\mathrm{I}$, et al. Analyzing incomplete longitudinal clinical trial data. Biostatistics 2004;5:445-64.

53. McKinney RE, Beskow LM, Ford DE, et al. Use of altered informed consent in pragmatic clinical research. Clin Trials 2015;12:494-502.

54. HHS.gov. Office for Human Research Protections (OHRP). 45 CFR 46. 2016 https://www.hhs.gov/ohrp/regulations-and-policy/ regulations/45-cfr-46/index.html (accessed 16 Aug 2018).

55. Lantos JD, Wendler D, Septimus E, et al. Considerations in the evaluation and determination of minimal risk in pragmatic clinical trials. Clin Trials 2015;12:485-93.

56. Suffoletto B, Lynch M, Pacella CB, et al. The effect of a statewide mandatory prescription drug monitoring program on opioid prescribing by emergency medicine providers across 15 hospitals in a single health system. J Pain 2018;19:430-8.

57. ICMJE. Recommendations | Defining the Role of Authors and Contributors. http://www.icmje.org/recommendations/browse/rolesand-responsibilities/defining-the-role-of-authors-and-contributors. html (accessed 8 Mar 2019). 